\title{
Physical Mesh Data Structures
}

\author{
Ergun Akleman* \\ Departments of Visualization \& \\ Computer Science and Engineering \\ Texas A\&M University
}

\author{
Shenyao Ke \\ $\&$ You Wu \\ Department of Visualization \\ Texas A\&M University
}
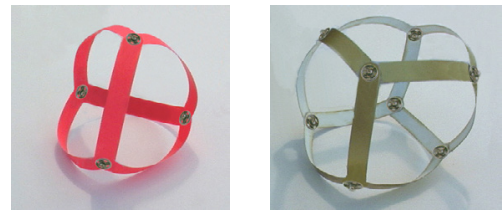

(a) Graphs Embedded on Surfaces
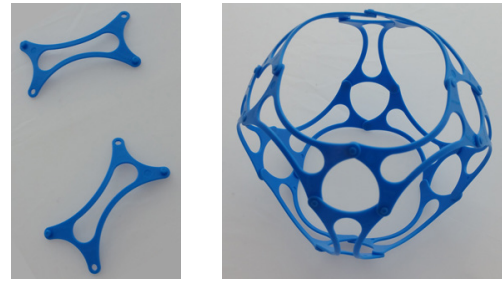

(d) Flexeez: Quad-Edge
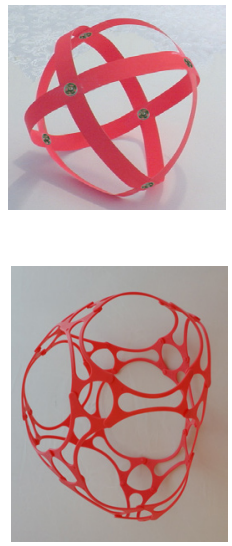
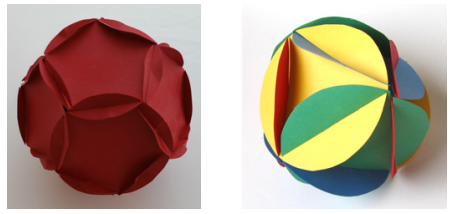

(c) Half-Edge Structures

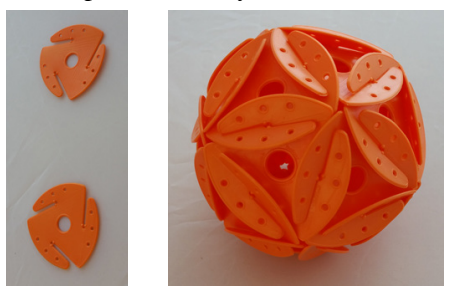

(e) Space Chips: Half-Edge
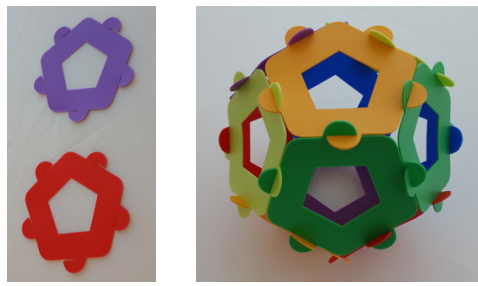

(f) It's Phun: Half-Edge

Figure 1: The top row shows examples of our polyhedral sculptures that correspond to physical versions of mesh data structures. The bottom row shows examples of geometric construction toys that correspond to quad-edge and half-edge data structures. For geometric toys, we show both individual elements and examples of constructed shapes.

\section{Abstract}

In this work, we demonstrate that existing mesh data structures in computer graphics can be used to categorize physical polygonal models. Based on this categorization, we develop a system to unfold any polygonal mesh based on widely used mesh-data structures. Using our system, any shape can be constructed by using laser-cut developable panels based on one of the existing mesh data structures. This categorization is also useful for the creation and classification of geometric toys. It is particularly useful to develop new construction methods for complicated sculptural and architectural shapes.

\section{Keywords: Shape Modeling and Construction}

Concepts: •Mathematics of computing $\rightarrow$ Graphs and surfaces; Geometric topology;

\section{Introduction and Motivation}

The need for categorization is partially motivated by recent geometric models and toys developed to construct topological properties of any Platonic and Archimedean polyhedron (see Figure 1). We observed that these toys and models corresponds to well-known

*e-mail:ergun.akleman@gmail.com

Permission to make digital or hard copies of part or all of this work for personal or classroom use is granted without fee provided that copies are not made or distributed for profit or commercial advantage and that copies bear this notice and the full citation on the first page. Copyrights for thirdparty components of this work must be honored. For all other uses, contact the owner/author(s). (c) 2016 Copyright held by the owner/author(s).

SIGGRAPH '16, July 24-28, 2016, Anaheim, CA,

ISBN: 978-1-4503-4282-7/16/07

DOI: http://dx.doi.org/10.1145/2897839.2927462 mesh data structures such as half-edge and quad-edge data. It was straightforward to generalize these ideas to any polygonal meshes with planar faces. Based on this point of view, we have developed a mesh unfolding system that can produce planar panels for each one of these physical data structures. The sculptures shown in Figures 2 and 3 are constructed using unfolded panels created using our system.

All orientable manifold-mesh data structures are based on the graph rotation systems (GRS), which is a powerful tool for guaranteeing topological consistency [Gross and Tucker 2001]. A graph embedded in an orientable surface corresponds to a rotation system, namely, the one in which the rotation at each vertex is consistent with the cyclic order of the neighboring vertices in the embedding [Gross and Tucker 2001]. GRS has explicitly been used as a physical mesh data structure as shown in Figures 1(b) and 2 to construct large shapes [Xing et al. 2011; Hernandez et al. 2013]. GRS have also been implicitly used in computer graphics for representing and manipulating orientable (and non-orientable) 2-manifold surfaces in the guise of various data structures, such as half-edges [Mäntylä 1988], quad-edges [Guibas and Stolfi 1985], winged-edges [Baumgart 1972].
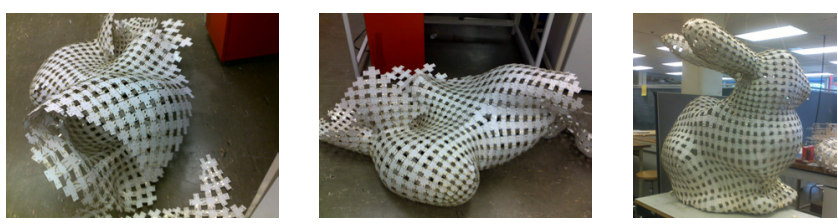

Figure 2: Construction of a large bunny using phsyical version of graphs rotation systems (GRS). 


\section{Categorization}

Graph Rotation Systems : One of the implications of GRS is that these physical mesh data structures can be considered instances of thickened graphs embedded on orientable 2-manifold surfaces. The graph $G$ can be "thickened" in the surface $S$ by enlarging each vertex of $G$ to a small polygonal disk called a vertex-band and widening each edge to a narrow rectangular edge-band that joins the vertex-bands at its endpoints [Akleman et al. 2010].. The union of the vertex-bands and the edge-bands is topologically a surface $T$ with boundary, called a thickening of the graph $G$, and also called a band decomposition. Figure 1(a) shows paper strip sculptures that are used to represent graphs embedded surfaces [Akleman et al. 2010]. (a)

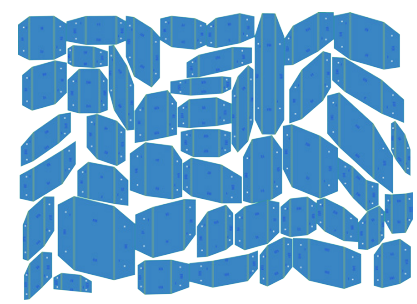

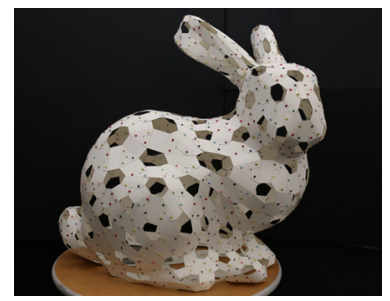

(b)
Figure 3: Examples of models that corresponds to quad-edge mesh data structures. (a) shows a set of quad-edge panels packed into a single page and (b) shows a large bunny constructed by using such quad-edge panels.

Half-Edge Data Structures : Although a variety of physical versions of half-edge structures are possible, all existing physical versions of half-edge data structures that we know are some type of slitted structures, in which slitted parts play the role of connector (i.e. half-edge) [Akleman et al. 2016]. Slitted structures were first introduced by George Hart as slide-together structures for platonic solids [Hart 2004]. Jace Miller independently discovered a general version by showing the slitted polygons can be used to construct any physical shape as interlocking-slitted structure [Miller and Akleman 2008]. Figures 1(e) and (f) show two types of recently discovered "slitted" geometric toys that correspond to half-edge data structures.

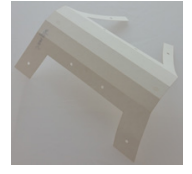

(a)

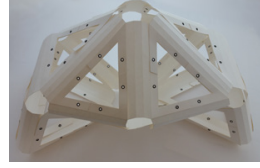

(b)

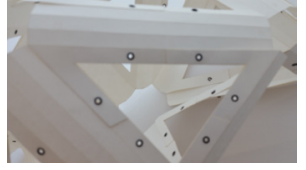

(c)
Figure 4: Examples of models that corresponds to winged-edge mesh data structures. (a) shows a bended single panel, (b) shows a winged-edge sculpture and (c) shows detail of the sculpture.

Quad-Edge Data Structures : The concept of quad-edge emerges from the fact that the edges are the only standard elements in a 2-manifold mesh consisting of two edge-ends and two half-edges [Guibas and Stolfi 1985]. Any edge can, therefore, be thickened always to a rectangular edge-band. Figure 1(d) shows a geometri construction toy, called Flexeez in USA, that corresponds to quad-edge data structure. David Reimann independently discovered another physical version of quad-edge data structures by demonstrating that any Platonic and Archimedean polyhedron can be constructed using only squares cut from anisotropic materials [Reimann 2015]. We have developed a system to unfold any planar polygonal mesh into quad panels and build large sculptures as shown in Figure 3 [Akleman et al. 2016]. One advantage of quad-edges for construction is that we only have to use cylindrical quad shaped panels that can be cut using anisotropic materials such as veneer [Akleman et al. 2016]. Another advantage of quad-edge panels is that they can pack very well and can be printed using less material.

Winged-Edge Data Structures : As far as we know, there is no physical counter part of winged-edge data structure, which is one of the oldest formalized data structures that supports manifold surfaces [Baumgart 1972]. We expected because of its wings, winged edges can provide a strong structure. However, as soon as we build one, we realized that it is hard to put together. Another problem is that winged-edge panels do not pack well.

Conclusion: Based on our analysis, the best way to construct large shapes seems to be physical versions of quad-edge data structures. Quad-edge panels have three advantages over others: (1) They pack densely without leaving much of unused material, (2) Every connection is made by only one other panel, which makes connection thickness uniform, and (3) They can be build using anisotropic materials providing extra stability. This work was partially supported by the National Science Foundation under Grants NSFEFRI-1240483, NSF-CMMI-1548243 and NSF-ECCS-1547075.

\section{References}

Akleman, E., Chen, J., AND Gross, J. L. 2010. Paper-strip sculptures. In Shape Modeling International Conference (SMI), 2010, IEEE, 236-240.

Akleman, E., Wu, Y., Ke, S., Borhani, A., Kalantar, N., AND CHEN, J. 2016. Construction with physical version of quad-edge data structures. Computers \& Graphics (accepted).

BAUMGART, B. G. 1972. Winged edge polyhedron representation. Tech. rep., Technical Report, Stanford University.

Gross, J. L., AND TUCKeR, T. W. 2001. Topological graph theory. Courier Dover Publications.

Guibas, L., AND STOLFI, J. 1985. Primitives for the manipulation of general subdivisions and the computation of voronoi. $A C M$ Transactions on Graphics (TOG) 4, 2, 74-123.

HART, G. 2004. slide-together: Geometric paper constructions. In Teachers' workshop at Bridges Conference.

Hernandez, E. A. P., Hu, S., Kung, H. W., Hartl, D., And AKLEMAN, E. 2013. Towards building smart self-folding structures. Computers \& Graphics 37, 6, 730-742.

MÄNTYLÄ, M. 1988. An introduction to solid modeling. Computer Science Press.

Miller, J., AND AKleman, E. 2008. Edge-based intersected polyhedral paper sculptures constructed by interlocking slitted planar pieces. In Bridges Leeuwarden: Mathematics, Music, Art, Architecture, Culture, Tarquin Publications, 259-264.

REIMANN, D. A. 2015. Nonplanar expansions of polyhedral edges in platonic and archimedean solids. In Proceedings of Bridges 2015: Mathematics, Music, Art, Architecture, Culture, Tessellations Publishing, Phoenix, Arizona, 143-150.

Xing, Q., Esquivel, G., AKleman, E., Chen, J., And Gross, J. 2011. Band decomposition of 2-manifold meshes for physical construction of large structures. In ACM SIGGRAPH 2011 Posters and Talks, ACM, 58. 